\title{
Ischemic postconditioning improves the expression of cellular membrane connexin 43 and attenuates the reperfusion injury in rat acute myocardial infarction
}

\author{
HUA HE ${ }^{1}$, NAN $^{2}{ }^{2}$, ZHIHONG ZHAO ${ }^{3}$, FUSHENG HAN ${ }^{1}$, XIFU WANG ${ }^{1}$ and YUJIE ZENG ${ }^{1}$ \\ ${ }^{1}$ Department of Emergency Cardiology, Beijing Anzhen Hospital, Capital Medical University; \\ ${ }^{2}$ Department of Cardiology, China-Japan Friendship Hospital, Beijing 100029; ${ }^{3}$ Department of Cardiology, \\ Pudong New Area District Zhoupu Hospital, Shanghai 201318, P.R. China
}

Received April 5, 2015; Accepted May 21, 2015

DOI: 10.3892/br.2015.485

\begin{abstract}
To investigate the effects of cellular membrane connexin $43(\mathrm{Cx} 43)$ and the potential details in ischemic postconditioning (IPOC)-induced cardioprotection, ischemia/reperfusion (IR) models were generated in 8-week-old male Sprague-Dawley rats by ligating the left coronary artery anterior descending branch. The serum levels of myocardial creatases, nitric oxide (NO) and malondialdehyde (MDA) levels, infarct size, arrhythmia events, expression and distribution of $\mathrm{Cx} 43$, ultrastructure and apoptosis in the myocardium in different treatments with IR, IR + IPOC, IR + diazoxide or IR + IPOC + 5-hydroxydecanoate acid (5-HD) were investigated . Consequently, IPOC decreased infarct size (10.9 vs. 43.3\%, $\mathrm{P}<0.01)$ and the levels of myocardial creatases, NO and MDA, and improved the expression (16.8 vs. $25.2 \%$ and 6.4 vs. $32.8 \%$, after 1- and 3-h reperfusion, respectively; $\mathrm{P}<0.01)$ and distribution of $\mathrm{Cx} 43$, ultrastructure and apoptosis (19.2 vs. $42.9 \%$, $\mathrm{P}<0.01)$ significantly. Diazoxide partly simulated the effects, and 5-HD attenuated but not completely abolished the effects of IPOC. In addition, the phosphorylated Cx43 (p-Cx43) level in the IR + IPOC group was lower than that in the IR + diazoxide group after 1-h reperfusion (26.1 vs. 29.4\%, $\mathrm{P}>0.05$ ); however, it was reversed after 3 -h reperfusion and the $\mathrm{p}-\mathrm{Cx} 43$ level in the IR + IPOC group was significantly higher than that in the IR + diazoxide group ( 32.8 vs. $18.7 \%, \mathrm{P}<0.01)$. In conclusion, cell membrane $\mathrm{Cx} 43$ is also involved in the process of IPOC-induced cardioprotection and the improvement of membrane $\mathrm{Cx} 43$ is more dependent on mitochondrial $\mathrm{K}_{\mathrm{ATP}}$ in the earlier phase of IPOC compared to the late phase of IPOC.
\end{abstract}

Correspondence to: Dr Yujie Zeng, Department of Emergency Cardiology, Beijing Anzhen Hospital, Capital Medical University, 2 Anzhen Road, Beijing 100029, P.R. China

E-mail: zengyujie0722@163.com

Key words: ischemia/reperfusion, postconditioning, membrane connexin 43

\section{Introduction}

Ischemic heart diseases are leading causes of fatality worldwide, and irreversible and widespread loss of myocardial cells and subsequent ventricular remodeling induced by acute myocardial infarction are the main elements resulting in chronic heart failure and permanent loss of labor force (1). There are 17.3 million people who succumbed of ischemic heart diseases in 2008 and the number is estimated to be 23.6 million by 2030 (2). Revascularization could improve the prognosis of the patients with acute myocardial infarction (1), however, reperfusion could induce additional injury, which is sometimes extremely severe or fatal and diminishes the benefits of revascularization. Basic studies (3-6) and small-sample clinical data $(7,8)$ have verified that ischemic postconditioning (IPOC) could attenuate injury induced by ischemia/reperfusion (IR) and certain studies (8-10) have indicated that the mitochondrial $\mathrm{K}_{\text {ATP }}\left(\right.$ mitoK $_{\text {ATP }}$ ) channel plays an important role in cardioprotection effects of IPOC, but its molecular mechanisms are not completely clear thus far. Connexin 43 (Cx43; a gap junction protein located mainly in the ventricular myocardium in mammals) plays an important role in intercellular electrical conduction and cell survival, but whether the changes of membrane Cx43 are involved in IPOC-induced cardioprotective effects and the corresponding details is unclear. Therefore, exploring the effects and mechanisms could aid the understanding of the deeply molecular mechanisms of IPOC, thus providing information for constructing an effective strategy to enhance cardioprotection.

Therefore, the present study was performed to investigate the changes of membrane $\mathrm{Cx} 43$, cellular ultrastructure, infarct size, reperfusion arrhythmia in different states of IR and IPOC to determine potential mechanisms and associated details of IPOC.

\section{Materials and methods}

Study approval. The study was reviewed and approved by the Institutional Ethics Committee of the Anzhen Hospital (Beijing, China) and Beijing Institute of Heart, Lung and Blood Vessel Diseases on Animal Resource (Beijing, China), 
and conformed to the guiding principles of 'Guide for the Care and Use of Laboratory Animals' (National Institutes of Health Publication no. 83-23, revised 1996) during, maintaining and using the animals.

Animal model and experiment protocol. All the rats were randomly divided into 5 groups to receive different treatments ( $n=16$ in each group): i) Sham; ii) IR group, 30-min ischemia followed by reperfusion for $180 \mathrm{~min}$; iii) IR + IPOC group, after the 30-min ischemia, IPOC was performed through application of 5 intermittent cycles of 10 -sec reperfusion and 10-sec ischemia immediately followed by reperfusion for $178 \mathrm{~min}$ $20 \mathrm{sec}$; iv) IR + diazoxide group, diazoxide (30 $\mu \mathrm{mol} / \mathrm{l})$ was administrated intravenously in the first $10 \mathrm{~min}$ of reperfusion; and v) IR + IPOC + 5-hydroxydecanoate acid (5-HD) group, 5 -HD $(300 \mu \mathrm{mol} / \mathrm{l})$ was administrated intravenously in the first $10 \mathrm{~min}$ of reperfusion.

The IR model was prepared with 8-week-old male Sprague-Dawley rats weighing 260-310 g (Laboratory Animal Division, Medicine Department, Beijing University, Beijing, China), as described previously (11). The sternum was incised and the heart was exposed subsequent to anesthetizing with $1 \%$ pentobarbital $(40 \mathrm{mg} / \mathrm{kg}$ body weight) through peritoneal injection and support from a breathing machine. The left coronary artery anterior descending branch was found and marked with silk and ligated with ligator to establish the rat IR model (baseline electrocardiography was recorded at $10 \mathrm{~min}$ after marking with silk). The coronary arteries of the rats in the sham group were only marked and not ligated.

Occurrence of arrhythmia in the different groups. Electrocardiograms of the rats in the different groups at baseline and during the experiment were monitored continuously (electrocardiograph 2E31A; SAN-EI TECH Ltd., Chiba, Japan) and arrhythmia events were recorded.

Measurement of myocardial creatases, nitric oxide (NO) and malondialdehyde (MDA) in the different groups. The blood was obtained from abdominal aorta immediately after 3-h reperfusion, and the serum levels of NO, MDA, creatine kinase (CK), CK-MB and lactate dehydrogenase (LDH) in the different groups were measured with assay kits (Jiancheng Technology Co., Ltd., Nanjing, China) according to the manufacturer's instructions.

Evaluation of infarct size in the different groups. Myocardial infarct size was measured as described previously (12). After having finished reperfusion, the left anterior descending artery was reoccluded and Evans blue dye was administered intravenously to stain the normal region of the left ventricle (LV) and the heart was rapidly excised. LV tissue was isolated and cut into 10 cross-sectional pieces of equal thickness. The non-stained LV area at risk (AAR) was separated from the surrounding blue-stained LV normal zone and the two regions were separately incubated at $37^{\circ} \mathrm{C}$ for $15 \mathrm{~min}$ in $1 \%$ triphenyltetrazolium chloride (TTC) in $0.1 \mathrm{M}$ phosphate buffer adjusted to $\mathrm{pH}$ 7.4. The tissues were fixed overnight in $10 \%$ formaldehyde. AAR and blue-stained LV normal zone regions were weighed for determination of AAR/LV. TTC stains living tissue a deep red color, but necrotic tissue is TTC-negative and appears white within the AAR slices. Each slice was scanned with a commercial scanner (Canoscan LiDE 60; Canon Inc., Tokyo, Japan) and infarct and non-infarct areas were measured using an image analysis program (Image-Pro Plus Version 6.0; Media Cybernetics, Georgia, MD, USA). Myocardial infarct size was expressed as a percentage of the AAR.

Western blot analysis of membrane Cx43 expression in the different groups. The rats were sacrificed respectively at 1- and 3-h after reperfusion by intraperitoneal injection of $3 \%$ pentobarbital sodium with the dose of $100 \mathrm{mg} / \mathrm{kg}$, their hearts were taken immediately and LV tissues below the ligation point were obtained and prepared. The samples were homogenized in Protein Extraction reagents (Pierce 78510; Pierce Biotechnology, Inc., Waltham, MA, USA) and harvested in $50 \mu 1$ of sample buffer, boiled and sonicated. Protein lysates were separated on $10 \%$ sodium dodecyl sulphate-polyacrylamide gel electrophoresis. Following transfer to the polyvinylidene fluoride membranes and blocking with skimmed milk $(5 \% \mathrm{w} / \mathrm{v})$, the blots were incubated with primary antibodies of Cx43 and GAPDH (C8093; Sigma-Aldrich, St. Louis, MO, USA; and G5262; Sigma, Santa Clara, CA, USA, respectively) overnight at $4^{\circ} \mathrm{C}$. GAPDH served as the internal reference. Primary antibody binding was detected with an enhanced chemiluminescence western blotting kit (Amersham Biosciences, Piscataway, NJ, USA) and quantified by laser densitometry using the Typhoon 9400 fluorescent scanner together with ImageQuant 5.0 software (Amersham Biosciences) as previously described (13).

Immunohistochemistry analysis of membrane CX43 in the different groups. Left ventricular tissue below the ligation point was fixed with $10 \%$ formaldehyde solution at 1- and 3-h after reperfusion, respectively, embedded in epoxy resin and sectioned, and subsequently processed with the immunohistochemistry kit (HaoYang Bioscience Co., Tianjing, China) according to the manufacturer's instructions (anti-Cx43 antibody was purchased from Santa Cruz Biotechnology, Inc., Dallas, TX, USA; sc-271837), stained with diaminobenzidine (DAB) and counterstained with hematoxylin. Subsequently, the samples were observed with a microscope (BX51; Olympus, Tokyo, Japan).

Ultrastructure of the myocardial cell. The ultrastructure of the myocardial cell was observed with a transmission electron microscope as described previously (14). For electron microscopic examination, following fixation in glutaraldehyde, the sample was refixed in $1 \%$ osmium tetroxide, embedded in epoxy resin, sectioned at $0.1-\mu \mathrm{m}$ and double-stained with uranium acetate and lead citrate. The sections were observed with a transmission electron microscope (Hitachi-600; Hitachi High-Technologies Corp., Tokyo, Japan).

Apoptosis analysis in the myocardium in the different groups. The tissue sample in each group was embedded in paraffin, sectioned at $4-\mu \mathrm{m}$, blocked with $3 \%$ hydrogen peroxide and incubated for $60 \mathrm{~min}$ with terminal deoxynucleotidyl transferase dUTP nick end labeling reaction mixture (45 $\mu 1$ Enzyme solution $+405 \mu \mathrm{l}$ Label solution) at $37^{\circ} \mathrm{C}$ in the dark. Subsequently, the sample was stained with converter-peroxidase solution 
and DAB substrate, and counterstained with hematoxylin. Apoptosis in each group was analyzed by observing with a light microscope (Motic BA400; Motic, Xiamen, China) and the apoptosis index was calculated.

Statistical analysis. All the values are expressed as mean \pm standard error of the mean. Differences in continuous variables between 2 groups were analyzed via the Student's t-test and differences between $\geq 3$ groups were evaluated via one-way analysis of variance with Bonferroni correction; differences in categorical data were assessed by the $\chi^{2}$ test, and in the case of low cell counts $(<5)$ the Fisher's exact test was used instead of $\chi^{2}$ test. $\mathrm{P}<0.05$ was considered to indicate a statistically significant difference.

\section{Results}

Various arrhythmias in different groups. No arrhythmia event occurred in the sham group and the occurrence rate of the arrhythmia events was $62.5,25.0,37.5$ and $50.0 \%$ in the IR, IR + IPOC, IR + diazoxide and IR + IPOC + 5-HD groups, respectively. There was a significant difference between the groups (Table I). The arrhythmias that happened during IR included ventricular premature beat (coupled rhythm), ventricular tachycardia, atrioventricular block and ventricular fibrillation (Fig. 1).

Levels of creatases, NO and MDA in the different groups. After 3-h reperfusion, the serum levels of CK, CK-MB, LDH, $\mathrm{NO}$ and MDA increased significantly in the IR group. IPOC and diazoxide impaired the increases, and 5-HD attenuated, but did not completely abolish, the effects of IPOC. There was no clear difference in the levels of NO and MDA between the IR + IPOC and IR + diazoxide groups (51.7 vs. $45.5 \mu \mathrm{mol} / 1$ and 6.93 vs. $6.55 \mathrm{nM}$, respectively; P>0.05), but the levels of $\mathrm{CK}$ and $\mathrm{CK}-\mathrm{MB}$ in the IR + diazoxide group was higher than that in the IR + IPOC group (3,286.9 vs. 3,775.9 U/1 and 670.2 vs. $747.3 \mathrm{U} / 1$, respectively; $\mathrm{P}<0.05$ ) (Table II).

Infarct size in the different groups. After 3-h reperfusion, the infarct size was $43.3 \pm 9.7 \%$ in the IR group, and IPOC and diazoxide decreased the size (10.9 and 15.5 vs. $43.3 \%$, respectively; $\mathrm{P}<0.05)$, and 5-HD attenuated, but not completely abolished, the effects of IPOC (37.7 vs. $43.3 \%, \mathrm{P}<0.05)$. The infarct size in the IR + IPOC group was smaller than that in the IR + diazoxide group, but this was not significant (Table III).

Membrane Cx43 expression in the different groups after 1- and 3-h reperfusion. Total $\mathrm{Cx} 43$ ( $\mathrm{t}-\mathrm{Cx} 43$ ) and phosphorylated $\mathrm{Cx} 43$ (p-Cx43) in the IR group decreased, and in particular, p-Cx43 decreased significantly (42.7 vs. $16.8 \%$ and 38.1 vs. $6.4 \%$, after 1 - and 3 -h reperfusion respectively, $\mathrm{P}<0.01)$. IPOC and diazoxide partly restored the levels of $\mathrm{t}-\mathrm{Cx} 43$ and $\mathrm{p}-\mathrm{Cx} 43$, and 5-HD alleviated, but not completely abolished, the effects of IPOC (16.8 vs. $21.7 \%$ and 6.4 vs. $14.2 \%$, after 1- and 3-h reperfusion, respectively, $\mathrm{P}<0.05)$. The $\mathrm{p}-\mathrm{Cx} 43$ level in the IR + IPOC group was lower than that in the IR + diazoxide group after 1-h reperfusion (26.1 vs. $29.4 \%, \mathrm{P}>0.05$ ), however, it was reversed after 3 -h reperfusion and the p-Cx43 level in the IR + IPOC group was significantly higher than that in the IR + diazoxide
Table I. Occurrence of arrhythmia in the different groups.

\begin{tabular}{|c|c|c|c|c|c|c|}
\hline \multirow[b]{2}{*}{ Group } & \multirow[b]{2}{*}{ No. } & \multirow{2}{*}{$\begin{array}{l}\text { During } \\
\text { ischemia, } \\
\text { times }\end{array}$} & \multicolumn{3}{|c|}{$\begin{array}{l}\text { During } \\
\text { reperfusion, } \\
\text { times }\end{array}$} & \multirow{2}{*}{$\begin{array}{c}\text { Occurence rate of } \\
\text { arrhythmia during } \\
\text { reperfusion, \% }\end{array}$} \\
\hline & & & $1 \mathrm{~h}$ & $2 \mathrm{~h}$ & $3 \mathrm{~h}$ & \\
\hline A & 16 & 0 & 0 & 0 & 0 & 0.0 \\
\hline B & 16 & 18 & 12 & 2 & 0 & $62.5^{\mathrm{a}, \mathrm{b}}$ \\
\hline $\mathrm{C}$ & 16 & 17 & 6 & 1 & 0 & $25.0^{\mathrm{a}, \mathrm{c}}$ \\
\hline $\mathrm{D}$ & 16 & 19 & 8 & 1 & 0 & $37.5^{\mathrm{a}-\mathrm{c}}$ \\
\hline $\mathrm{E}$ & 16 & 19 & 9 & 1 & 0 & $50.0^{\mathrm{a}-\mathrm{d}}$ \\
\hline
\end{tabular}

A, sham group; B, IR group; C, IR + IPOC group; D, IR + diazoxide group; E, IR + IPOC + 5-HD group. IR, ischemia/reperfusion; IPOC, ischemic postconditioning; 5-HD, 5-hydroxydecanoate acid. ${ }^{\mathrm{a}} \mathrm{P}<0.01$ vs. group $\mathrm{A} ;{ }^{b} \mathrm{P}<0.05$ vs. group $\mathrm{C} ;{ }^{\mathrm{c}} \mathrm{P}<0.01$ vs. group $\mathrm{B}$; ${ }^{\mathrm{d}} \mathrm{P}<0.05$ vs. group $\mathrm{D}$.

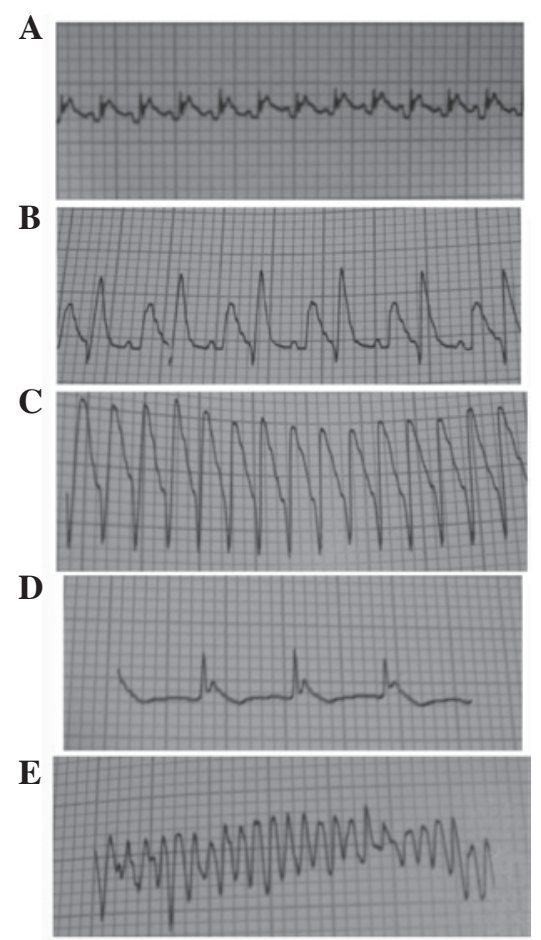

Figure 1. Electrocardiography in lead II at baseline, during ischemia and reperfusion. (A) Normal electrocardiography in lead II at baseline. (B-E) Ventricular premature beat (coupled rhythm), ventricular tachycardia, atrioventricular block and ventricular fibrillation in lead II, respectively, presented during ischemia and reperfusion in each group.

group (32.8 vs. $18.7 \%, \mathrm{P}<0.01)$, which suggested that mitoK $\mathrm{ATP}_{\mathrm{P}}$ plays an important role in the expression of $\mathrm{Cx} 43$ during the early phase of IR. IPOC still significantly restored the expression of $\mathrm{Cx} 43$ through other pathways besides opening mitoK $\mathrm{K}_{\mathrm{ATP}}$ during the late phase of IR and the benefit of single opening mitoK $_{\text {ATP }}$ was limited in the late phase of IR (Figs. 2 and 3).

Distribution of membrane CX43 in the different groups after $3-h$ reperfusion. In the sham group, membrane Cx43 was 
Table II. Serum levels of NO, MDA, CK, CK-MB and LDH in the different groups.

\begin{tabular}{lrlcccr}
\hline Group & No. & NO, $\mu \mathrm{mol} / 1$ & MDA, nM & CK, U/l & CK-MB, U/l & LDH, U/l \\
\hline A & 14 & $26.0 \pm 9.7$ & $3.88 \pm 0.88$ & $896.3 \pm 125.5$ & $214.6 \pm 87.7$ & $260.4 \pm 98.6$ \\
B & 9 & $86.1 \pm 29.0^{\mathrm{a}}$ & $11.08 \pm 3.22^{\mathrm{a}}$ & $5,283.9 \pm 1,348.2^{\mathrm{a}}$ & $985.1 \pm 223.0^{\mathrm{a}}$ & $1,135.2 \pm 327.6^{\mathrm{a}}$ \\
C & 13 & $51.7 \pm 12.6^{\mathrm{b}, \mathrm{c}}$ & $6.93 \pm 1.77^{\mathrm{b}, \mathrm{c}}$ & $3,286.9 \pm 949.8^{\mathrm{b}-\mathrm{d}}$ & $670.2 \pm 237.6^{\mathrm{b}-\mathrm{d}}$ & $780.7 \pm 249.1^{\mathrm{b}, \mathrm{c}}$ \\
D & 12 & $45.5 \pm 12.9^{\mathrm{b}, \mathrm{c}}$ & $6.55 \pm 2.46^{\mathrm{b}, \mathrm{c}}$ & $3,775.9 \pm 1,034.6^{\mathrm{b}, \mathrm{c}, \mathrm{e}}$ & $727.3 \pm 304.4^{\mathrm{b}, \mathrm{e}, \mathrm{e}}$ & $813.4 \pm 278.3^{\mathrm{b}, \mathrm{c}}$ \\
E & 10 & $70.8 \pm 14.5^{\mathrm{b}, \mathrm{d}, \mathrm{e}}$ & $8.69 \pm 1.47^{\mathrm{b}, \mathrm{d}, \mathrm{e}}$ & $4,633.3 \pm 1,177.3^{\mathrm{b}, \mathrm{d}, \mathrm{e}}$ & $886.8 \pm 275.4^{\mathrm{b}, \mathrm{d}, \mathrm{e}}$ & $1,002.8 \pm 376.7^{\mathrm{d}, \mathrm{e}}$ \\
\hline
\end{tabular}

A, sham group; B, IR group; C, IR + IPOC group; D, IR + diazoxide group; E, IR + IPOC + 5-HD group. IR, ischemia/reperfusion; IPOC, ischemic postconditioning; 5-HD, 5-hydroxydecanoate acid; NO, nitric oxide; MDA, malondialdehyde; CK, creatine kinase; LDH, lactate dehydrogenase. ${ }^{a} \mathrm{P}<0.01$ vs. group $\mathrm{A} ;{ }^{\mathrm{b}} \mathrm{P}<0.05$ vs. group $\mathrm{B} ;{ }^{\mathrm{c}} \mathrm{P}<0.05$ vs. group $\mathrm{E}$; ${ }^{\mathrm{d}} \mathrm{P}<0.05$ vs. group $\mathrm{D} ;{ }^{\text {e }} \mathrm{P}<0.05$ vs. group $\mathrm{C}$.

Table III. Infarct size in the different groups.

\begin{tabular}{lcc}
\hline Group & No. & Infarct size, $\%$ \\
\hline A & 4 & $0.0 \pm 0.0$ \\
B & 4 & $43.3 \pm 9.7^{\mathrm{a}}$ \\
C & 4 & $10.9 \pm 6.3^{\mathrm{a}-\mathrm{c}}$ \\
D & 4 & $15.5 \pm 9.5^{\mathrm{a}-\mathrm{c}}$ \\
E & 4 & $37.7 \pm 6.7^{\mathrm{a}, \mathrm{b}, \mathrm{d}, \mathrm{e}}$ \\
\hline
\end{tabular}

A, sham group; B, IR group; C, IR + IPOC group; D, IR + diazoxide group; E, IR + IPOC + 5-HD group. IR, ischemia/reperfusion; IPOC, ischemic postconditioning; 5-HD, 5-hydroxydecanoate acid. ${ }^{\mathrm{a}} \mathrm{P}<0.01$ vs. group $\mathrm{A} ;{ }^{b} \mathrm{P}<0.05$ vs. group $\mathrm{B} ;{ }^{c} \mathrm{P}<0.05$ vs. group $\mathrm{E}$; ${ }^{\mathrm{d}} \mathrm{P}<0.05$ vs. group $\mathrm{C}$; ${ }^{\text {e }}<0.05$ vs. group $\mathrm{D}$.
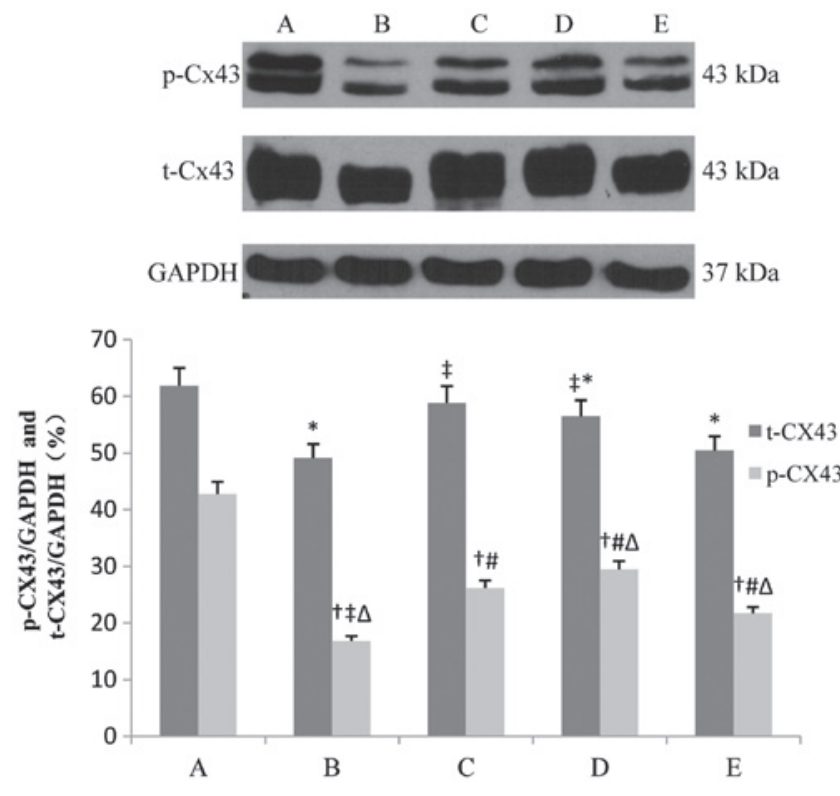

Figure 2. Expression of t- $\mathrm{Cx} 43$ and $\mathrm{p}-\mathrm{Cx} 43$ in each group after 1 -h reperfusion. Bar graphs showing the relative expression of $\mathrm{t}-\mathrm{Cx} 43$ and p-Cx43 over GAPDH following densitometric scanning for western blot analysis. Data are represented as mean \pm standard deviation for 3 different experiments. Lanes A-E are the sham, IR, IR + IPOC, IR + diazoxide and IR + IPOC + 5-HD groups, respectively. Cx43, connexin 43; t-Cx43, total Cx43; p-Cx43, phosphorylated Cx43; IR, ischemia/reperfusion; IPOC, ischemic postconditioning; 5-HD, 5-hydroxydecanoate acid. ${ }^{*} \mathrm{P}<0.05$ vs. t-Cx43 in group $\mathrm{A} ;{ }^{\dagger} \mathrm{P}<0.05$ vs. $\mathrm{p}-\mathrm{Cx} 43$ in group $\mathrm{A} ;{ }^{\dagger} \mathrm{P}<0.05$ vs. $\mathrm{t}-\mathrm{Cx} 43$ in group $\mathrm{B}$; ${ }^{\#} \mathrm{P}<0.05$ vs. $\mathrm{p}-\mathrm{C} \times 43$ in group $\mathrm{B} ;{ }^{\wedge} \mathrm{P}<0.05$ vs. $\mathrm{p}-\mathrm{Cx} 43$ in group $\mathrm{C}$.
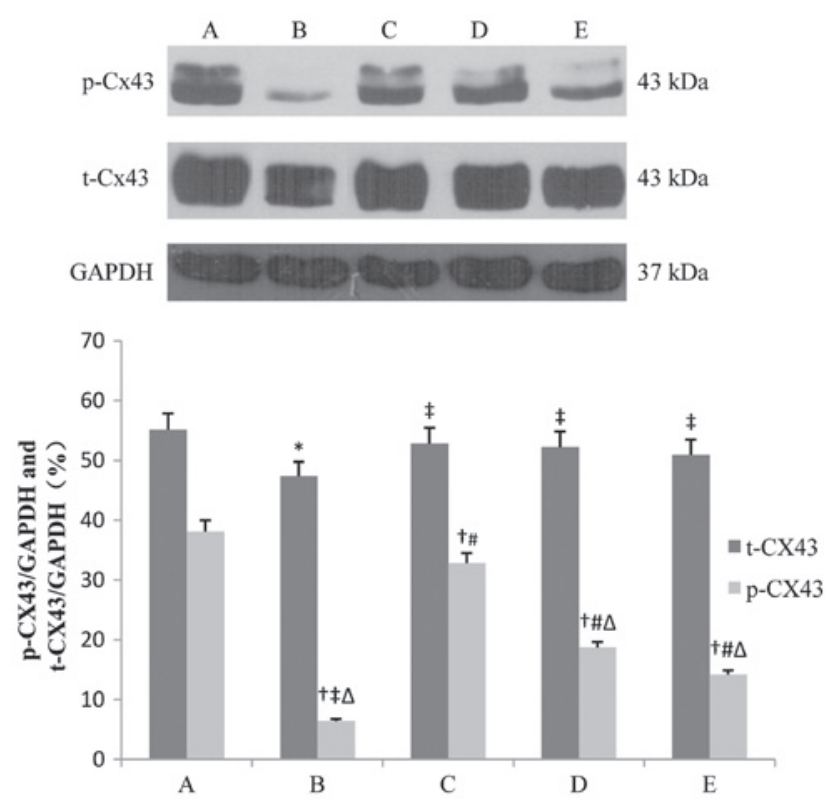

Figure 3. Expression of t-Cx43 and p-Cx43 in each group after $3-\mathrm{h}$ reperfusion. Bar graphs showing the relative expression of $\mathrm{t}-\mathrm{Cx} 43$ and p-Cx43 over GAPDH following densitometric scanning for western blot analysis. Data are represented as mean \pm standard deviation for 3 different experiments. Lanes A-E are the sham, IR, IR + IPOC, IR + diazoxide and IR + IPOC + 5-HD groups, respectively. Cx43, connexin 43; t-Cx43, total Cx43; p-Cx43, phosphorylated Cx43; IR, ischemia/reperfusion; IPOC, ischemic postconditioning; 5-HD, 5-hydroxydecanoate acid. ${ }^{*} \mathrm{P}<0.05$ vs. t-Cx43 in group $\mathrm{A} ;{ }^{\dagger} \mathrm{P}<0.05$ vs. $\mathrm{p}-\mathrm{Cx} 43$ in group $\mathrm{A} ;{ }^{\stackrel{ }{ }} \mathrm{P}<0.05$ vs. $\mathrm{t}-\mathrm{Cx} 43$ in group $\mathrm{B}$; ${ }^{\#} \mathrm{P}<0.05$ vs. $\mathrm{p}-\mathrm{Cx} 43$ in group $\mathrm{B} ;{ }^{\wedge} \mathrm{P}<0.05$ vs. $\mathrm{p}-\mathrm{Cx} 43$ in group $\mathrm{C}$.

located mainly in the intercalated disc between proximate myocardial cells and there was hardly any $\mathrm{Cx} 43$ at the cell lateral membrane. After ischemia and 3-h reperfusion, Cx43 was distributed on the whole surface of the cells, and was not limited in the intercalated disc. The structure of the intercalated disc was unclear. $\mathrm{Cx} 43$ was also redistributed, but the majority of them were still located in the intercalated disc in the IR + IPOC group. There was also $\mathrm{Cx} 43$ at the cell lateral membrane, but $\mathrm{Cx} 43$ was mainly in the intercalated disc. The intercalated disc was sparse in the IR + diazoxide group. There was significant redistribution of $\mathrm{Cx} 43$ and less $\mathrm{Cx} 43$ in the disc in the IR + IPOC + 5-HD group (Fig. 4).

Changes of the ultrastructures of myocardial cells after 3-h reperfusion. In the sham group, the structure of the intercalated 


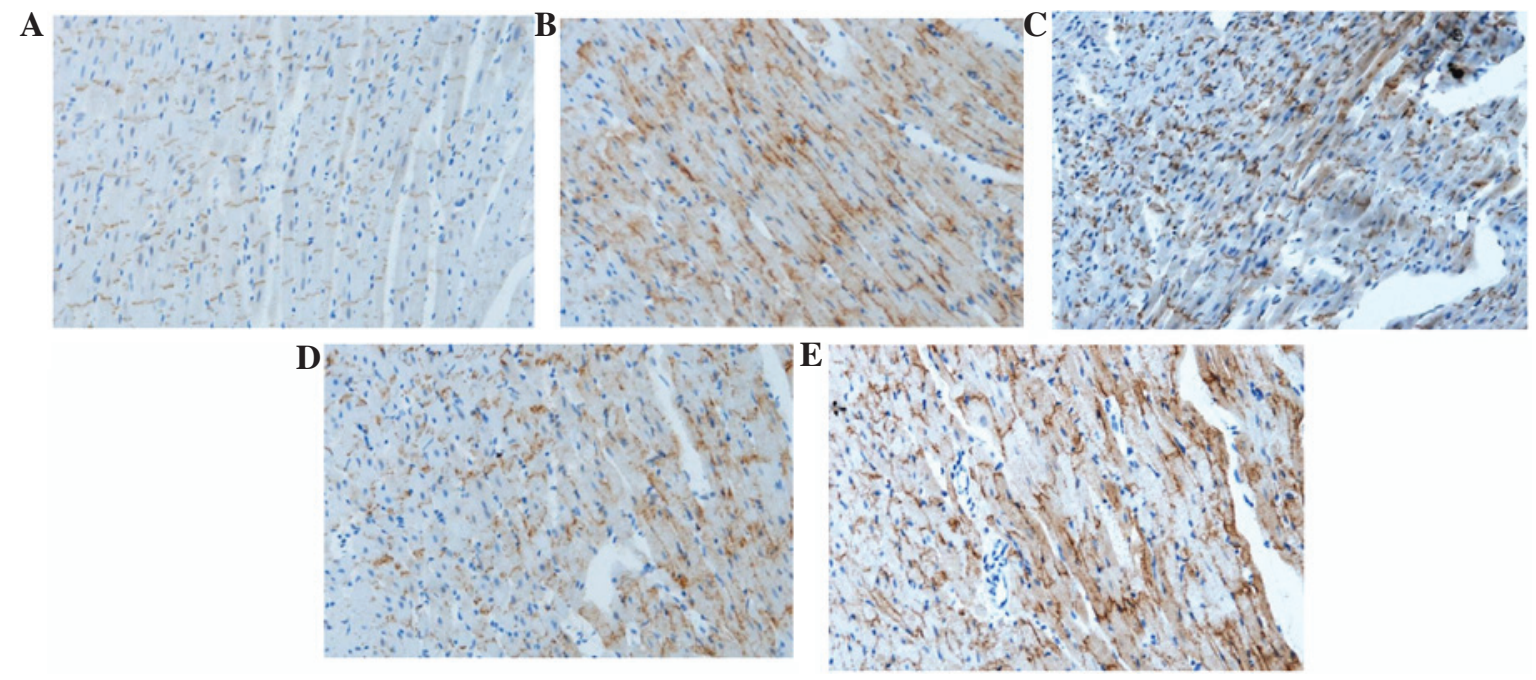

Figure 4. Distribution of Cx43 in each group after 3-h reperfusion. Blue indicates the cell nucleus. These pictures indicate that IR resulted in distribution of Cx43 (brown) on the whole cell surface (not only limited in the intercalated disc) and clear construction of the intercalated disc. IPOC attenuated the redistribution and the majority of $\mathrm{Cx} 43$ was located in the junction along the length of the neighboring myocardial cells. Diazoxide also improved the distribution of $\mathrm{Cx} 43$, whereas the majority of $\mathrm{Cx} 43$ was redistributed in the IR + IPOC + 5-HD group, which indicated that 5-HD impaired the effects of IPOC on Cx43. (A) Sham, (B) IR, (C) IR + IPOC, (D) IR + diazoxide and (E) IR + IPOC + 5-HD groups. Cx43, connexin 43; IR, ischemia/reperfusion; IPOC, ischemic postconditioning; 5-HD, 5-hydroxydecanoate acid.

A

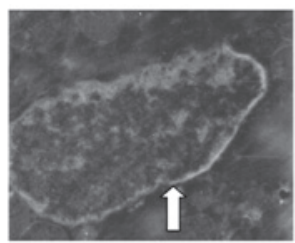

B

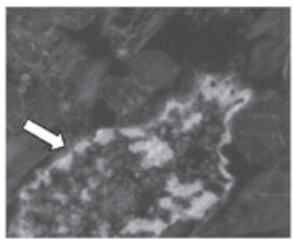

C

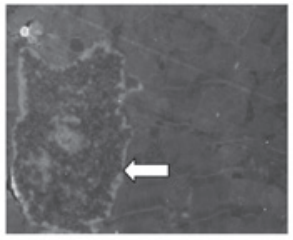

D

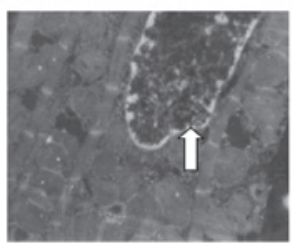

$\mathbf{E}$

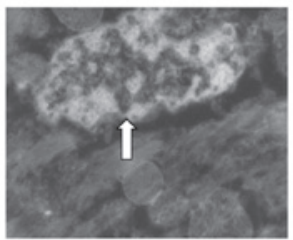

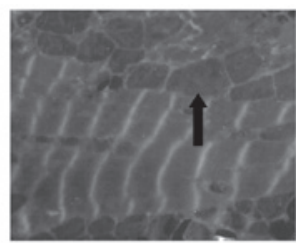
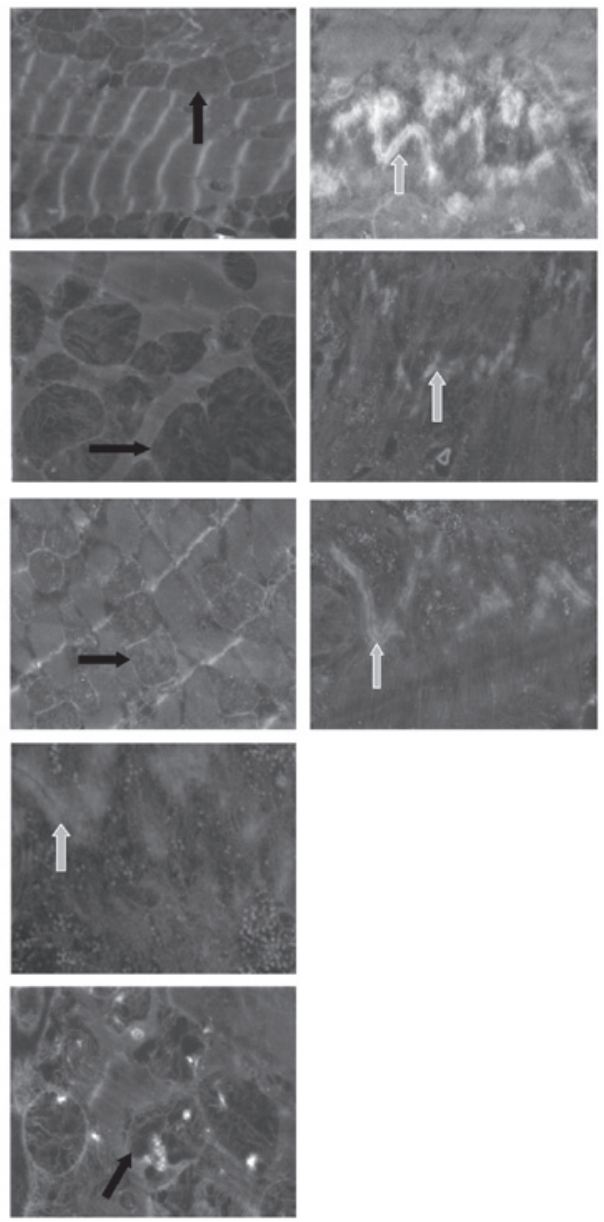

Figure 5. Ultrastructure of the myocardial cell in each group. Cell nucleus is indicated by the white arrow, mitochondrion by the black arrow and intercalated disc by the gray arrow. These pictures indicate that the structure of the intercalated disc was clear and there was a regular array of myofilaments, echelonment of $\mathrm{Z}$ line and normal mitochondrion in the sham group. There was a disrupted and textureless intercalated disc, dissolved myocardial cells and condensed nucleus and megamitochondrion or the mitochondrion with a vacuole in the IR group. IPOC significantly attenuated the pathological changes induced by IR and the majority of the disc had an intact structure. Diazoxide also alleviated the changes, but there were only fewer discs in which their structures were clear. 5-HD largely impaired the IPOC-induced improvement in ultrastructure and there were disrupted myofilaments, flocculent mitochondrion and the structure of the disc was not intact. (A) Sham, (B) IR, (C) IR + IPOC, (D) IR + diazoxide and (E) IR + IPOC + 5-HD groups. IR, ischemia/reperfusion; IPOC, ischemic postconditioning; 5-HD, 5-hydroxydecanoate acid. 


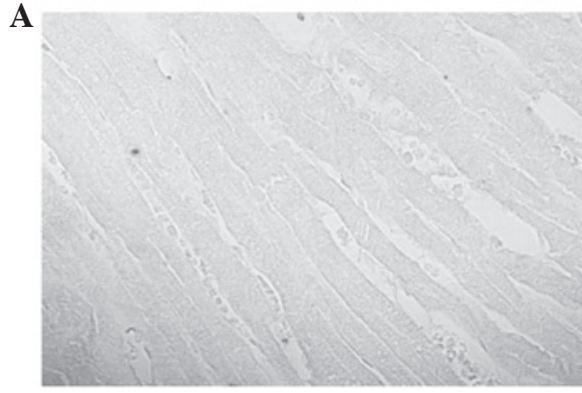

D

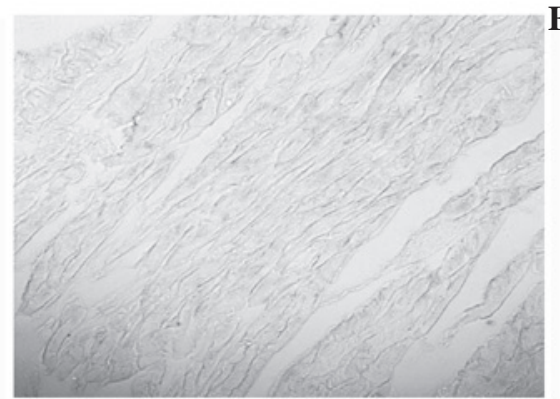

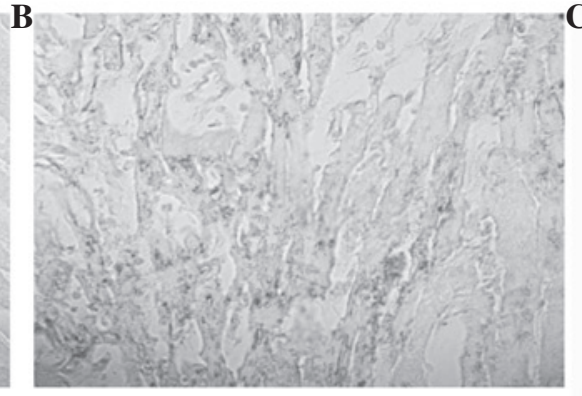

$\mathbf{E}$

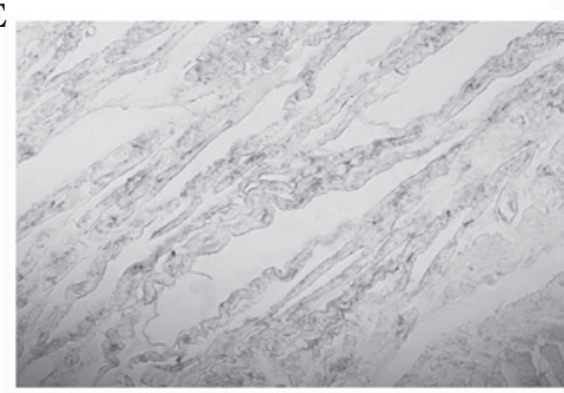

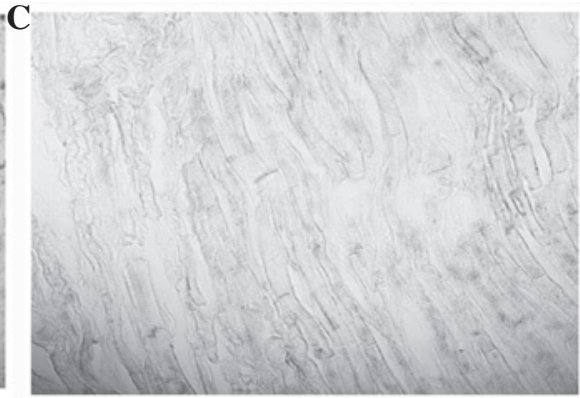

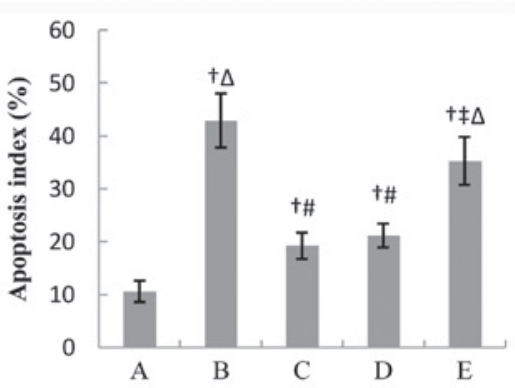

Figure 6. Apoptosis in the myocardium in each group after 3-h reperfusion. There were numerous apoptotic cells (black) and clearly disrupted myocardial fibers in the IR group. IPOC attenuated the apoptosis and disorderly array of myocardial cells. The apoptosis in the IR + diazoxide group was similar to the IR + IPOC group. 5-HD attenuated the effects of IPOC, but did not completely abolish the effects of IPOC. The apoptosis index in the IR + IPOC + 5-HD group was still lower than that in the IR group. The bar graph shows the relative apoptosis index in each group. Data are represented as mean \pm standard deviation for 5 different experiments; apoptosis index $=$ (apoptosis cell counts/total cell counts) $x 100 \%$. Apoptosis cell nucleus is shown as black. (A) Sham, (B) IR, (C) IR + IPOC, (D) IR + diazoxide and (E) IR + IPOC + 5-HD groups. IR, ischemia/reperfusion; IPOC, ischemic postconditioning; 5-HD, 5-hydroxydecanoate acid. ${ }^{\mathrm{P}} \mathrm{P}<0.01$ vs. group $\mathrm{A} ;{ }^{*} \mathrm{P}<0.05$ vs. group $\mathrm{B} ;{ }^{*} \mathrm{P}<0.01$ vs. group $\mathrm{B} ;{ }^{\wedge} \mathrm{P}<0.05$ vs. group $\mathrm{C}$.

disc was clear and there was a regular array of myofilaments, echelonment of $\mathrm{Z}$ line and normal mitochondrion. After ischemia and 3-h reperfusion, there was a disrupted and textureless intercalated disc, dissolved myocardial cells and condensed nucleus and megamitochondrion or the mitochondrion with a vacuole. IPOC attenuated the pathological changes significantly and the majority of the disc had an intact structure. Diazoxide also alleviated the changes induced by IR, but there were only fewer discs whose structures were clear. 5-HD largely impaired the IPOC-induced improvement in ultrastructure and there were disrupted myofilaments, flocculent mitochondrion and the structure of the disc was not intact (Fig. 5).

Apoptosis in the different groups after 3-h reperfusion. There were numerous black apoptotic cells and evidently disrupted myocardial fibers in the IR group. IPOC attenuated the apoptosis significantly $(19.2$ vs. $42.9 \%, \mathrm{P}<0.01)$ and there was a relatively regular array of myocardial cells. Following treatment with diazoxide, the apoptosis was similar to the IR + IPOC group. 5-HD attenuated the effects of IPOC and there was more significant apoptosis and disorderly myocardial array in the IR + IPOC + 5-HD group than that in the IR + IPOC group. The effects of IPOC were not abolished completely, and the apoptosis index in the IR + IPOC + 5-HD group was still lower than that in the IR group (33.2 vs. $42.9 \%, \mathrm{P}<0.05)$ (Fig. 6).

\section{Discussion}

The present study investigated the expression and distribution of Cx43, ultrastructure and apoptosis in the myocardium. The levels of myocardial creatases, NO and MDA, infarct size and arrhythmia events for the different treatments of
IR, IR + IPOC, IR + diazoxide or IR + IPOC + 5-HD were assessed. The results indicated that IPOC decreased infarct size and the levels of myocardial creatases, NO and MDA, and improved the expression and distribution of $\mathrm{Cx} 43$, ultrastructure and apoptosis. Diazoxide could partly simulate the effects, and 5-HD attenuated, but not completely abolished, the effects of IPOC, which suggested that cell membrane Cx43 is also involved in the process of IPOC-induced cardioprotection. The improvement of membrane $\mathrm{Cx} 43$ is much more dependent on mitoK $\mathrm{ATP}_{\text {in }}$ the earlier phase of IPOC than that in the late phase of IPOC, and to the best of our knowledge, this is the first report of this.

Previous data have indicated that IPOC could induce cardioprotection by inhibiting the expression of matrix metalloproteinase-2 (15), decreasing no-reflow (8), inhibiting apoptosis (16) and attenuating inflammatory response (5), and certain studies indicated that mitoK $_{\text {ATP }}$ plays a crucial role in IPOC-induced cardioprotection $(9,10,17)$. The present study indicated that IPOC decreased infarct size and the levels of myocardial creatases, improved the expression and distribution of Cx43 and apoptosis. Opening mitoK ${ }_{\text {ATP }}$ with diazoxide partly simulated the effects of IPOC, and an inhibitor of mitoK $_{\text {ATP }}, 5-\mathrm{HD}$, attenuated, but not abolished completely, the effects of IPOC, which suggested that mitoK ${ }_{\text {ATP }}$ plays an important role. However, there are other independent key pathways in IPOC-induced cardioprotection.

Cx43, a gap junction protein located mainly in ventricular myocardium in mammals, plays an important role in intercellular electric conduction and cell survival. Previous studies have indicated that mitochondrial $\mathrm{Cx} 43$ may be involved in

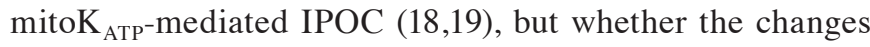
of membrane $\mathrm{Cx} 43$ are involved in cardioprotection of 
mitoK $_{\text {ATP-mediated IPOC and the corresponding details }}$ are not clear. The present study indicated that IR decreased expression of t-Cx43 and p-Cx43 (particularly the latter) and induced the redistribution of $\mathrm{Cx} 43$ on the cell surface, and IPOC attenuated the decreases. Diazoxide partly restored the levels of $\mathrm{Cx} 43$, and 5-HD alleviated the effects of IPOC. Therefore, mitoK $_{\text {ATP }}$ plays a more important role in expression of Cx43 during the earlier phase of IR, whereas the benefit of single opening mitoK $\mathrm{ATP}_{\mathrm{AT}}$ was limited in the late phase of IR.

Reperfusion injury is a problem in treatment for the patients with acute myocardial infarction and IPOC could attenuate the injury, but its mechanism is not clear. The present study indicated that cell membrane $\mathrm{Cx} 43$ is also involved in the process of IPOC-induced cardioprotection and the improvement of membrane $\mathrm{Cx} 43$ is much more dependent on mitoK $\mathrm{ATP}_{\mathrm{ATP}}$ in the earlier phase of IPOC. Therefore, it is important that appropriate drugs should be selected in different phases of IR to protect membrane $\mathrm{Cx} 43$ against injury.

In conclusion, cell membrane $\mathrm{Cx} 43$ is also involved in the process of IPOC-induced cardioprotection and the improvement of membrane $\mathrm{Cx} 43$ is much more dependent on mitoK ${ }_{\text {ATP }}$ in the earlier phase of IPOC than that in the late phase of IPOC.

The present study verified that cell membrane Cx43 is also involved in the process of IPOC-induced cardioprotection, and the detailed mechanisms of the changes of membrane $\mathrm{Cx} 43$ in IPOC would be a future task, thus find new potential targets for attenuating IR.

\section{Acknowledgements}

The present study was supported by the Development Funds of Capital Medicine (grant no. 2009-3022), Chinese Natural Science Foundation Grants (no. 81100142), Open Topic Funds Grants of Essential Laboratory in Cardiovasular Remodeling and Transforming Medicine, National Ministry of Education (no. 2010XGCS02), Cultivating Project Grants of Beijing for Highly Talented Men of Medicine (no. 2014-3-042), and the Superintendent Cultivating Funds Grants of Beijing Anzhen Hospital (no. 2010F03). The study was also supported by the Beijing Anzhen Hospital, Capital University of Medical Sciences and Beijing Institute of Heart Lung and Blood Vessel Diseases. The authors thank Dr TieMin Ma for his valuable suggestions regarding the rat IR model and Dr ZhuQing Jia for her assistance of the ultrastructure analysis with electron microscope.

\section{References}

1. Roger VL, Go AS, Lloyd-Jones DM, Benjamin EJ, Berry JD, Borden WB, Bravata DM, Dai S, Ford ES, Fox CS, et al; American Heart Association Statistics Committee and Stroke Statistics Subcommittee: Heart disease and stroke statistics - 2012 update: A report from the American Heart Association. Circulation 125: e2-e220, 2012.

2. Mendis S, Puska P and Norrving BO (eds): Global Atlas on Cardiovascular Disease Prevention and Control. World Health Organization, Geneva, 2011.
3. Jiang ZH, Zhang TT and Zhang JF: Protective effects of fasudil hydrochloride post-conditioning on acute myocardial ischemia/reperfusion injury in rats. Cardiol J 20: 197-202, 2013.

4. Wei C, Li H, Han L, Zhang L and Yang X: Activation of autophagy in ischemic postconditioning contributes to cardioprotective effects against ischemia/reperfusion injury in rat hearts. J Cardiovasc Pharmacol 61: 416-422, 2013.

5. Wang NP, Pang XF, Zhang LH, Tootle S, Harmouche S and Zhao ZQ: Attenuation of inflammatory response and reduction in infarct size by postconditioning are associated with downregulation of early growth response 1 during reperfusion in rat heart. Shock 41: 346-354, 2014.

6. Tu Y, Wan L, Fan Y, Wang K, Bu L, Huang T, Cheng Z and Shen B: Ischemic postconditioning-mediated miRNA-21 protects against cardiac ischemia/reperfusion injury via PTEN/Akt pathway. PLoS One 8: e75872, 2013.

7. Crimi G, Pica S, Raineri C, Bramucci E, De Ferrari GM, Klersy C, Ferlini M, Marinoni B, Repetto A, Romeo M, et al: Remote ischemic post-conditioning of the lower limb during primary percutaneous coronary intervention safely reduces enzymatic infarct size in anterior myocardial infarction: A randomized controlled trial. JACC Cardiovasc Interv 6: 1055-1063, 2013.

8. Mewton N, Thibault H, Roubille F, et al: Postconditioning attenuates no-reflow in STEMI patients. Basic Res Cardiol 108: $383,2013$.

9. Shimizu S, Oikawa R, Tsounapi P, Inoue K, Shimizu T, Tanaka K, Martin DT, Honda M, Sejima T, Tomita S, et al: Blocking of the ATP sensitive potassium channel ameliorates the ischaemia-reperfusion injury in the rat testis. Andrology 2: 458-465, 2014

10. Mykytenko J, Reeves JG, Kin H, Wang NP, Zatta AJ, Jiang R, Guyton RA, Vinten-Johansen $\mathbf{J}$ and Zhao ZQ: Persistent beneficial effect of postconditioning against infarct size: Role of mitochondrial K(ATP) channels during reperfusion. Basic Res Cardiol 103: 472-484, 2008.

11. Mukhopadhyay P, Mukherjee S, Ahsan K, Bagchi A, Pacher P and Das DK: Restoration of altered microRNA expression in the ischemic heart with resveratrol. PLoS One 5: e15705, 2010.

12. Ichinomiya T, Cho S, Higashijima U, Matsumoto S, Maekawa $T$ and Sumikawa K: High-dose fasudil preserves postconditioning against myocardial infarction under hyperglycemia in rats: Role of mitochondrial KATP channels. Cardiovasc Diabetol 11: 28, 2012.

13. Pogwizd SM, Qi M, Yuan W, Samarel AM and Bers DM: Upregulation of $\mathrm{Na}(+) / \mathrm{Ca}(2+)$ exchanger expression and function in an arrhythmogenic rabbit model of heart failure. Circ Res 85: 1009-1019, 1999.

14. Sui H, Wang W, Wang PH and Liu LS: Protective effect of antioxidant ebselen (PZ51) on the cerebral cortex of stroke-prone spontaneously hypertensive rats. Hypertens Res 28: 249-254, 2005.

15. Liu ZZ, Kong JB, Li FZ, Ma LL, Liu SQ and Wang LX: Ischemic postconditioning decreases matrix metalloproteinase-2 expression during ischemia-reperfusion of myocardium in a rabbit model: A preliminary report. Exp Clin Cardiol 18: e99-e101, 2013.

16. Sun H, Guo T, Liu L, Yu Z, Xu W, Chen W, Shen L, Wang J and Dou X: Ischemic postconditioning inhibits apoptosis after acute myocardial infarction in pigs. Heart Surg Forum 13: E305-E310, 2010.

17. Penna C, Mancardi D, Rastaldo R, Losano G and Pagliaro P: Intermittent activation of bradykinin $\mathrm{B} 2$ receptors and mitochondrial KATP channels trigger cardiac postconditioning through redox signaling. Cardiovasc Res 75: 168-177, 2007.

18. He Y,Zeng ZY,Zhong GQ, Li JY,Li WK and Li W: Mitochondrial connexin 43 and postconditioning protection in rabbits underwent myocardial ischemia/reperfusion injury. Zhonghua Xin Xue Guan Bing Za Zhi 38: 357-362, 2010 (In Chinese).

19. Rottlaender D, Boengler K, Wolny M, et al: Connexin 43 acts as a cytoprotective mediator of signal transduction by stimulating mitochondrial KATP channels in mouse cardiomyocytes. J Clin Invest 120: 1441-1453, 2010. 